\title{
Correction to: Mammalian Target of Rapamycin 2 (MTOR2) and C-MYC Modulate Glucosamine-6-Phosphate Synthesis in Glioblastoma (GBM) Cells Through Glutamine: Fructose-6-Phosphate Aminotransferase 1 (GFAT1)
}

\author{
Bo Liu ${ }^{1,2,3}$ (1) $\cdot$ Huan-Kai Yao ${ }^{3}$
}

Published online: 14 August 2019

(c) Springer Science+Business Media, LLC, part of Springer Nature 2019

\section{Correction to: \\ Cellular and Molecular Neurobiology (2019) 39:415-434 \\ https://doi.org/10.1007/s10571-019-00659-7}

The original version of this article unfortunately contained an error in author group. The authors Yi-Xiang See, Xin Chen, Zi-Kai Chen and Ze-Bin Huang were inadvertently included in the article.

The correct author group is presented with this erratum.

Publisher's Note Springer Nature remains neutral with regard to jurisdictional claims in published maps and institutional affiliations.

The original article can be found online at https://doi.org/10.1007/ s10571-019-00659-7.

Bo Liu

boliufhs@umac.edu.mo

1 Laboratory of Molecular Genetics, University of Maryland

School of Medicine, Baltimore 21021, USA

2 Department of Otorhinolaryngology Head \& Neck Surgery, University of Maryland School of Medicine, Baltimore 21021, USA

3 Faculty of Health Sciences, University of Macau, Hengqin 999078, Macau, China 